\title{
Night vision imaging systems development, integration and verification in military fighter aircraft
}

Sabatini, Roberto; Richardson, Mark; Cantiello, Maurizio; Toscano, Mario; Fiorini, Pietro; Zammit-Mangion, David; Jia, H

https://researchrepository.rmit.edu.au/esploro/outputs/9921859419901341/filesAndLinks?institution=61RMIT_INST\&index=null

Sabatini, R., Richardson, M., Cantiello, M., Toscano, M., Fiorini, P., Zammit-Mangion, D., \& Jia, H. (2013).

Night vision imaging systems development, integration and verification in military fighter aircraft. Journal of Aeronautics and Aerospace Engineering, 2(2), 1-12. https://doi.org/10.4172/2168-9792.1000106 Document Version: Published Version

Published Version: https://doi.org/10.4172/2168-9792.1000106

Repository homepage: https://researchrepository.rmit.edu.au (C) 2013 Sabatini $R$, et al

Downloaded On 2023/04/26 21:23:06 +1000 
Thank you for downloading this document from the RMIT Research Repository.

The RMIT Research Repository is an open access database showcasing the research outputs of RMIT University researchers.

RMIT Research Repository: http://researchbank.rmit.edu.au/

\section{Citation:}

Sabatini, R, Richardson, M, Cantiello, M, Toscano, M, Fiorini, P, Zammit-Mangion, D and Jia, H 2013, 'Night vision imaging systems development, integration and verification in military fighter aircraft', Journal of Aeronautics and Aerospace Engineering, vol. 2, no. 2, pp. 1-12.

See this record in the RMIT Research Repository at:

https://researchbank.rmit.edu.au/view/rmit:22671

Version: Published Version

\section{Copyright Statement:}

(C) 2013 Sabatini R, et al Creative Commons Attribution 4.0 International License.

\section{Link to Published Version:}

http://dx.doi.org/10.4172/2168-9792.1000106 


\title{
Night Vision Imaging Systems Development, Integration and Verification in MilitaryFighter Aircraft
}

\author{
Sabatini $\mathbf{R}^{1 *}$, Richardson $\mathrm{MA}^{2}$, Cantiello $\mathbf{M}^{3}$, Toscano $\mathbf{M}^{3}$, Fiorini $\mathbf{P}^{3}$, Zammit-Mangion $\mathrm{D}^{1}$ and $\mathrm{Jia}^{1}$ \\ ${ }^{1}$ Department of Aerospace Engineering, Cranfield University, Cranfield, Bedford, UK \\ ${ }^{2}$ Defence Academy of the United Kingdom, Shrivenham, Swindon, Cranfield University, UK \\ IItalian Ministry of Defense, Air Staff, Rome, Italy
}

\begin{abstract}
This paper describes the research and experimental flight testactivities conducted by the Italian Air Force Official Test Centre (RSV) in collaboration with Alenia S.p.A. and Cranfiled University, in order to confer the Night Vision Imaging Systems (NVIS) capability to the Italian TORNADO IDS (Interdiction and Strike) and ECR (Electronic Combat and Reconnaissance) aircraft. The activities included various Design, Development, Test and Evaluation (DDT\&E) activities, including Night Vision Goggles (NVG) integration, cockpit instruments and external lighting modifications, as well as various ground test sessions and a total of eighteen flight test sorties. RSV and Litton Precision Products were responsible of coordinating and conducting the installation activities of the internal and external lights. Particularly, an iterative process was established, allowing an in-site rapid correction of the major deficiencies encountered during the ground and flight test sessions. Both single-ship (day/night) and formation (night) flights were performed, shared between the Test Crews involved in the activities, allowing for a redundant examination of the various test items by all participants. An innovative test matrix was developed and implemented by RSV for assessing the operational suitability and effectiveness of the various modifications implemented. Also important was definition of test criteria for Pilot and Weapon Systems Officer (WSO) workload assessment during the accomplishment of various operational tasks during NVG missions. Furthermore, the specific technical and operational elements required for evaluating the modified helmets were identified, allowing an exhaustive comparative evaluation of the two proposed solutions (i.e., HGU-55P and HGU-55G modified helmets). The results of the activities were very satisfactory. The initial compatibility problems encountered were progressively mitigated by incorporating modifications both in the front and rear cockpits at the various stages of the test campaign. This process allowed a considerable enhancement of the TORNADO NVIS configuration, giving a good medium-high level NVG operational capability to the aircraft. Further developments also include the internal/external lighting for the Italian TORNADO "Mid Life Update" (MLU) and other programs, such as the AM-X aircraft internal/external lights modification/testing and the activities addressing low-altitude NVG operations with fast jets (e.g., TORNADO, AM$X, M B-339 C D$ ), a major issue being the safe ejection of aircrew with NVG and NVG modified helmets. Two options have been identified for solving this problem: namely the modification of the current Gentex HGU-55 helmets and the design of a new helmet incorporating a reliable NVG connection/disconnection device (i.e., a mechanical system fully integrated in the helmet frame), with embedded automatic disconnection capability in case of ejection. Other relevant issues to be accounted for in these new developments are the helmet dimensions and weight, the NVG usable FOV as a function of eye-relief distance, and helmet centre of gravity (moment arms) with and without NVG (impact on aircrew fatigue during training and real operational missions).
\end{abstract}

Keywords: Night vision imaging systems; Night vision goggles; NVG compatibility; Military avionics systems

\section{Introduction}

In recent years, the Italian Air Force (ITAF) set the requirements for Night Vision Imaging Systems (NVIS) to be integrated on TORNADOIDS (Interdiction and Strike version) and ECR (Electronic Combat and Reconnaissance version) aircraft for operational missions at medium and high altitudes.

The initial operational capability (operational certification for employment in peace-keeping operations) was achieved by RSV after a ground and flight test campaign (three ground sessions and six flight test sorties) conducted on modified aircraft interior and external lighting configurations, using the AN/AVS/9 (F4949) NVG manufactured by ITT-Night Vision. Successively, the full technical/formal process of avionics certification was undertaken under the direction of the Italian Ministry of Defense Aeronautical Armaments Certification Authority (Armaereo). The related flight test activities were conducted by the Italian Official Flight Test Centre with participation of the AleniaS.p.A. Flight Test Department. During the activity, Cranfield University provided technical advice regarding the mathematical models and analytical tools required for NVIS performance prediction and evaluation. The specific objectives of the TORNADO ground and flight test activities were the following:

- Internal and external lighting day and night evaluation with and without N/AVS/9 NVG (F4949)

- Workload assessment in single-ship and formation flights

*Corresponding author: Roberto Sabatini, Department of Aerospace Engineering, Cranfield University, Cranfield, Bedford, UK, Tel: +44 (0)1234 758290; Fax: +44 (0)1234 758203; E-mail: r.sabatini@cranfield.ac.uk

Received December 08, 2012; Accepted February 22, 2013; Published March 02, 2013

Citation: Sabatini R, Richardson MA, Cantiello M, Toscano M, Fiorini P, et al. (2013) Night Vision Imaging Systems Development, Integration and Verification in MilitaryFighter Aircraft. J Aeronaut Aerospace Eng 2: 106. doi:10.4172/21689792.1000106

Copyright: (c) 2013 Sabatini R, et al. This is an open-access article distributed under the terms of the Creative Commons Attribution License, which permits unrestricted use, distribution, and reproduction in any medium, provided the original author and source are credited. 
Citation: Sabatini R, Richardson MA, Cantiello M, Toscano M, Fiorini P, et al. (2013) Night Vision Imaging Systems Development, Integration and Verification in MilitaryFighter Aircraft. J Aeronaut Aerospace Eng 2: 106. doi:10.4172/2168-9792.1000106

- Ergonomic and operational evaluation of the HGU-55P and HGU-55G modified helmets

- N/AVS/9 NVG (F4949) cockpit stowage evaluation

- Determination of the TORNADO-NVIS combination resolution characteristics

- Determination, by ground tests and analysis, of the TORNADONVIS range performance

After brief overview of NVIS technology, this paper described the DDT\&E activities performed, with a special focus on cockpit design and ground/flight test methods developed and progressively refined throughout the activity.

\section{NVIS Technology Overview}

The Image Intensifier $\left(\mathrm{I}^{2}\right)$ is the core element of NVIS systems. $\mathrm{I}^{2}$ devices are electro-optic systems used to detect and intensify reflected energy in the visible and near infrared regions of the electromagnetic spectrum. They require some external illumination in order to operate because the image quality is a function of the reflective contrast. The performances of $\mathrm{I}^{2}$ devices are also dependant on atmospheric and environmental conditions. Particularly, penetration through moisture can be quite effective (especially when compared to other ElectroOptic (EO) devices, like FLIR systems), while smoke, haze and dust can significantly reduce $\mathrm{I}^{2}$ performance. Signal-to-noise ratio (SNR) is the parameter commonly used to characterize $\mathrm{I}^{2}$ systems performance.

Generation I (GEN I) NVG's were introduced into service in the mid 1960's during the Vietnam War. They used starlight scopes based on electron acceleration (i.e., no micro channel plates). Therefore, they were characterized by high power requirements and tube gains between 40,000 and 60,000. Multiple staging, required to increase gain, often determined an increase of image distortion, and the overall systems were large/heavy (i.e., not suitable for head mount). Furthermore, GEN I systems were very susceptible to booming and the MTBF of a typical GEN I NVG was in the order of about 10,000 hours.

Generation II (GEN-II) NVG's were introduced in the late 1960's and they were small enough to be head mounted. They used electron multiplication (i.e., micro channel plate-MCP), with increased tubes gain, reduced power requirements, and reduced size/weight. Furthermore, the new $\mathrm{I}^{2}$ technology reduced distortion and blooming (confined to specific MCP tubules halos). Typical GEN-II systems were the AN/PVS-5 ground system, and the AN/AVS-5A system modified for aircraft usage. The MTBF of typical GEN-II systems was in the order of about 2000-4000 hours (worst than GEN I), the tube gain was approximately 10,000 , and there was no inherent resolution improvement with respect to GEN I systems.

Improved photocathode performance, obtained by Gallium Arsenide (GaAs) components, determined a substantial improvement in spectral response with Generation III (GEN-III) systems. GEN-III matches night sky radiation better than GEN I and GEN-II systems, and can operate also in the absence of moon (starlight capability). Improved MCP performance were obtained by Aluminium Oxide coating, which decreases ion hits and increases MTBF (>10,000 hours). Today, GEN-III systems are widely used on most ground and in aircraft applications. Figure 1 shows the relative responses of the GEN-II/GENIII NVG systems and the human eye, together with the average night sky radiation $[1,2]$. The improvement obtained with GEN-III NVG systems is evident.

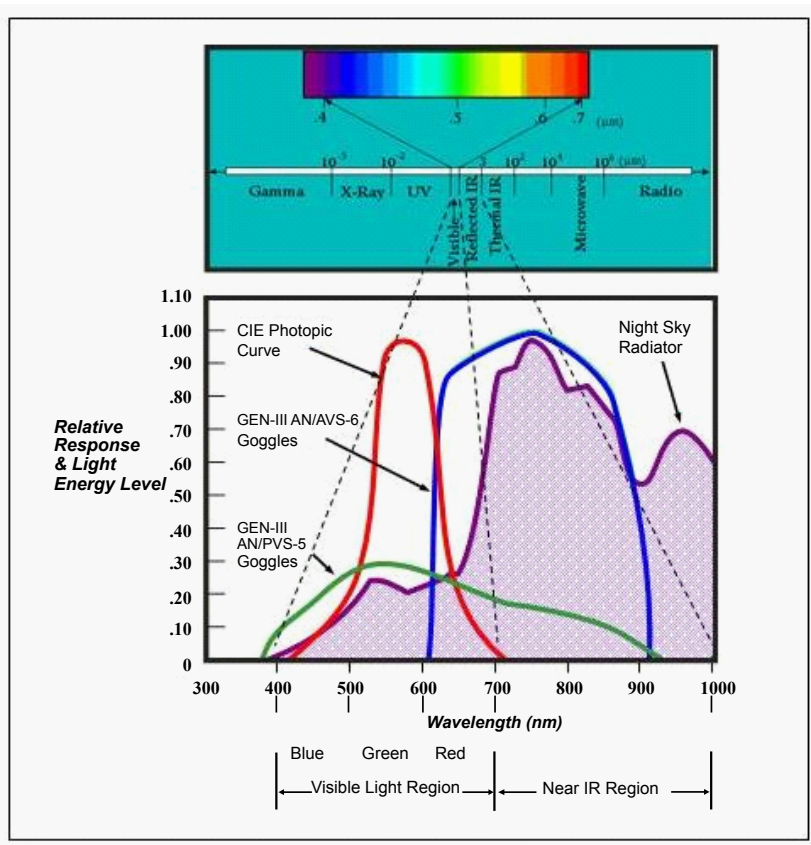

Figure 1: Relative responses of NVGs and the human eye.

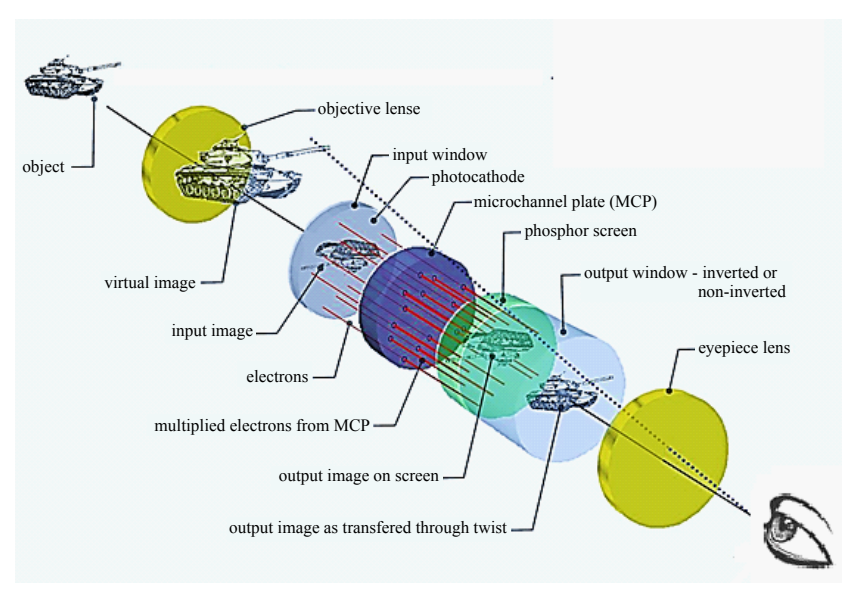

Figure 2: Architecture of an Image Intensifier.

As illustrated in figure 2 , an $\mathrm{I}^{2}$ device is typically composed by the following elements:

- Objective Lens

- Minus Blue Filter

- Photocathode

- Ion Barrier Film

- Microchannel Plate

- Phosphor Screen

- Image Inverter

- Eyepiece Lens

The Objective Lens combines the optical elements and focuses 
Citation: Sabatini R, Richardson MA, Cantiello M, Toscano M, Fiorini P, et al. (2013) Night Vision Imaging Systems Development, Integration and Verification in MilitaryFighter Aircraft. J Aeronaut Aerospace Eng 2: 106. doi:10.4172/2168-9792.1000106

incoming photons onto the photocathode (inverted image. In most airborne NVG's, the Objective Lens is coated with a "minus blue" filter (necessary for compatible cockpit lighting). It focuses from several inches to infinity (depending on NVG). Particularly, in airborne applications, infinity focusing is used in order to obtain:

\section{- NVG external viewing}

- Look Under/Around NVG for cockpit and instrument viewing

In airborne NVG's a "minus-blue" filter is coated inside the objective lens. Its purpose is to reject visible light and to prevent other specific wavelengths from entering the image intensifier. Therefore, it allows the use of properly emitting/filtered lighting to illuminate the cockpit for viewing underneath the goggles. There are three different classes of NVG objective lens filters:

- Class A: blocks below $625 \mathrm{~nm}$ (blue/green)

- Class B: blocks below $665 \mathrm{~nm}$ (blue/green/reduced red)-allows use of color displays

- Class C (leaky green)-incorporates notch cut-out to permit viewing of specific wavelength

The Photocathode (PC) converts light energy (photon) to electrical energy (electrons). The PC Inner surface is coated with a photosensitive material. Particularly, we list the following materials used in GEN-I/II and GEN-III systems:

- GEN-I/II: S-20 multi-alkali compound, sensitive between 400 and $850 \mathrm{~nm}$ (peak sensitivity at 500-600 nm);

- GEN-III: Gallium Arsenide (GaAs), sensitive from 600-900 nm (impact of photons cause release of electrons).

Typical PC responsivity figures are $250-550 \mu \mathrm{A} / \mathrm{lm}$ for GEN-II systems and 1,000-1,800 $\mu \mathrm{A} / \mathrm{lm}$ for GEN-III systems. As illustrated in figure 3, GEN-III $\mathrm{I}^{2}$ tubes are currently fabricated with a so called Ion Barrier (IB) film. This film extends tube life (protects the PC) but reduces the system performance (i.e., degrades signal-to-noise ratio).

The Microchannnel Plate (MCP) is a thin wafer (about $1 \mathrm{~mm}$ )

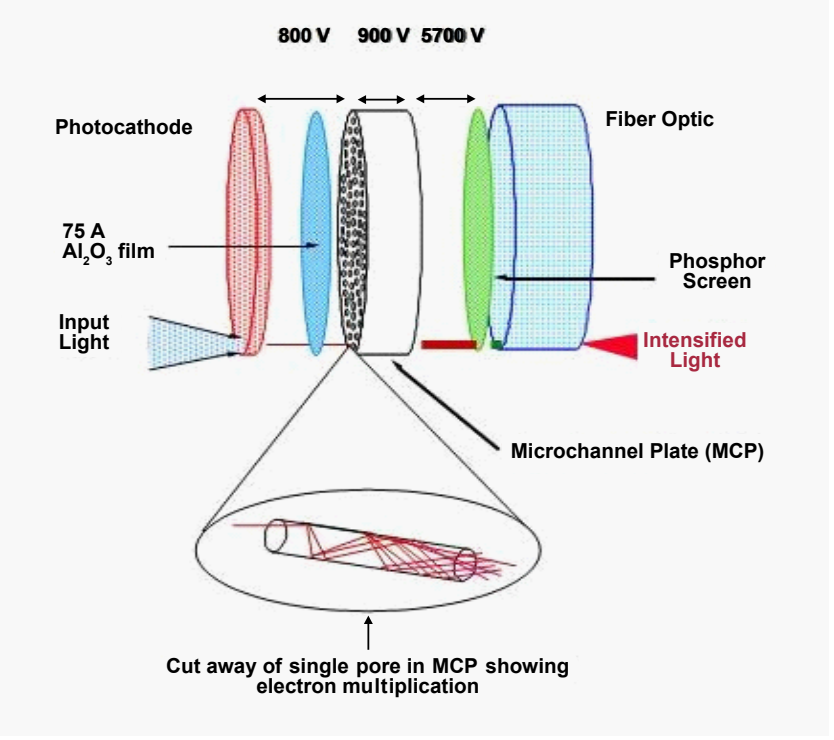

Figure 3: GEN-III I2 tube.

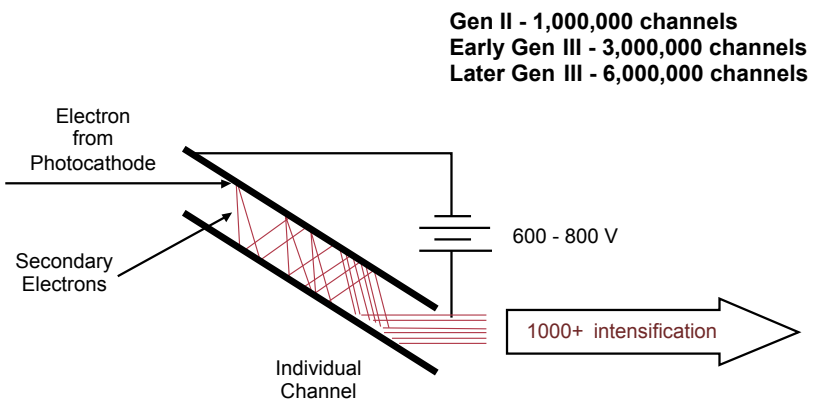

Figure 4: MCP working principle.

containing various millions of glass tubes or channels (typically 4-6 millions). Electrons from the PC enter the MCP tube (tube walls coated with lead compound rich in electrons) which is tilted (about 5 degrees) to ensure the impact of the electrons with the wall (Figure 4). When an electron impacts the tube wall, more electrons are released resulting in a cascade process. Electrons are then accelerated towards the phosphor by an electrical potential differential (positive pole at phosphor). The ultimate output is number of electrons and their velocity. Resolution is a function of number of MCP tubes.

The Phosphor Screen (PS) is a thin layer of phosphor at the output of the MCP. Phosphor emits light energy when struck by electrons (electro-luminescence). Light emitted by phosphor creates a visible (green) image.

The Image Inverter (INV) is a bundle of millions of light transmitting fibers. The bundle rotates 180 degrees to reorient the image (fiber optic twist). It also collimates image for correct positioning at the viewer's eye. Problems in INV manufacturing and installation result in adverse image effects, such as distortion and honeycomb appearance. Some NVG designs do not incorporate a fiber optic twist for reorienting the image.

The Eyepiece Lens (EL) is the final optical component of the NVG. It focuses the visible image on the retina of the viewer and, generally, a limited diopter adjustment is allowed to permit some correction for individual vision variations. In general, corrective lenses must still be worn by users (the system does not correct for astigmatism). Most GEN-II systems have a $15 \mathrm{~mm}$ eye-relief and a nominal $40^{\circ} \mathrm{FOV}$. GENIII systems typically have $25 \mathrm{~mm}$ nominal eye-relief which also provides the $40^{\circ} \mathrm{FOV}$ but enhances the ability to look under/around the NVG.

Signal to Noise Ratio (SNR) is a measure of image intensifier performance (resultant of the image intensification process). SNR for a NVG is defined as the ratio of electrons produced by ambient light (signal) to stray electrons (noise). Improved performance (larger SNR's) is produced by increasing the ambient light and/or improving the $\mathrm{I}^{2}$ (e.g., increasing PC sensitivity and decreasing the space between the elements)

\section{NVIS Compatibility Issues}

Intensified imagery of the outside scene is of primary importance to the aircrew. Incompatible light from cockpit sources and external lights are detected by the NVG and intensified, thus reducing the NVG gain. The resulting degraded image quality may not be readily apparent to the aircrew.

NVG compatible lighting results in instruments and displays being easily read with the unaided eye at night. However, all instruments must 
Citation: Sabatini R, Richardson MA, Cantiello M, Toscano M, Fiorini P, et al. (2013) Night Vision Imaging Systems Development, Integration and Verification in MilitaryFighter Aircraft. J Aeronaut Aerospace Eng 2: 106. doi:10.4172/2168-9792.1000106

still be readable during day. NVG compatible lighting is often invisible to the NVG, while "friendly" lighting may be visible to the goggles, but without changing the gain state of the goggle. Typically, NVG compatible instruments and displays only emit wavelengths to which the eye is most responsive (i.e., little red and no near-IR emission).

There are basically two different implementation methods which can be adopted for integrating NVG compatible lighting in the cockpit. These methods are the following

- Permanent lighting: Including integral instrument/display lighting, post and bezel lighting, food lighting using existing aircraft light fixtures or LED based light sources

- Temporary lighting: Including chemical light sticks and Light Emitting Diodes (LED) wiring harness

Also NVG compatible external lights have can be used in order to increase mission effectiveness, increase flight safety and decrease aircraft vulnerability (IR covert mode). Also in this case, there are basically two different approaches possible

- Introducing new equipment: Including conventional/filtered, electro-luminescent and LED technologies

- Retrofitting existing lights: Including filtering and modifying the existing light source

Another important aspect to be considered with NVIS compatible aircraft developments is the NVG-helmet integration. Particularly, the following are the main goals to be achieved:

- Reduce the NVG-helmet moment arms

- Reduce the weight

- Maximize usage of the available FOV (considering eye relief, exit pupil, etc.) visors)

- Allow use of various types of visors (including laser protection

\section{Description of Test Articles}

The test activities were carried out using the NVG mod. AN/AVS/9 F4949G (P/N 264359-8) produced by ITT-Night Vision (Figure 5). This is a GEN-III NVG, with class B filter and $40^{\circ}$ nominal Field-of-View (FOV).

The goggles were installed on both the Gentex HGU-55/G and HGU-55/P standard helmets, using the ITT Night Vision helmet modification kit NSN 5340-01-442-641 as illustrated in the figures 6 and 7.
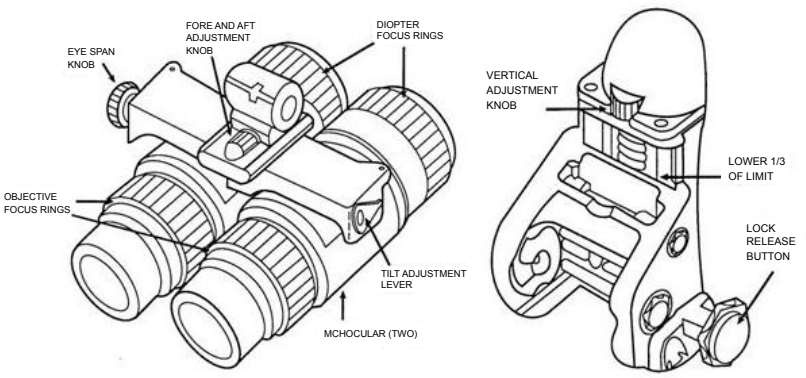

Figure 5: NVG mod.AN/AVS/9 F4949P.

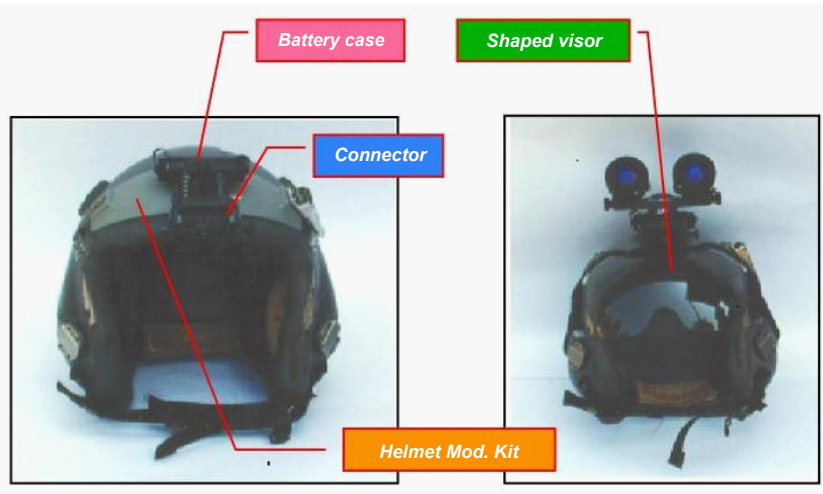

Figure 6: Modified HGU-55/P helmet with NVG installed.

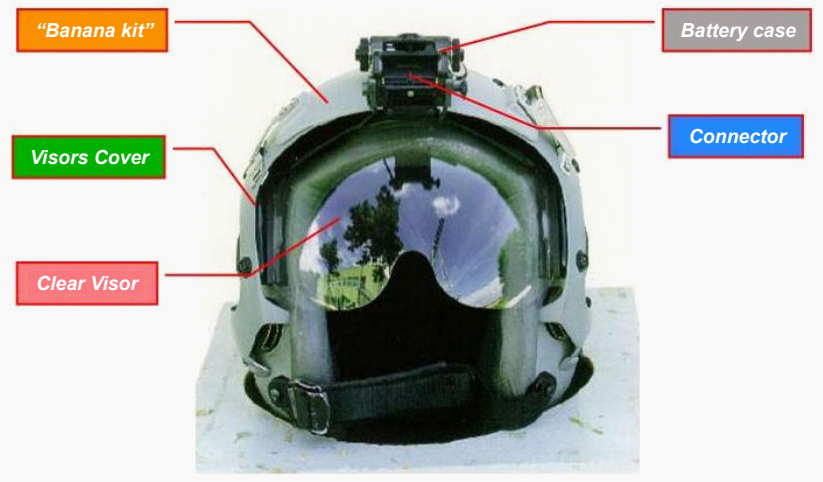

Figure 7: Modified HGU-55/G helmet with NVG installed.

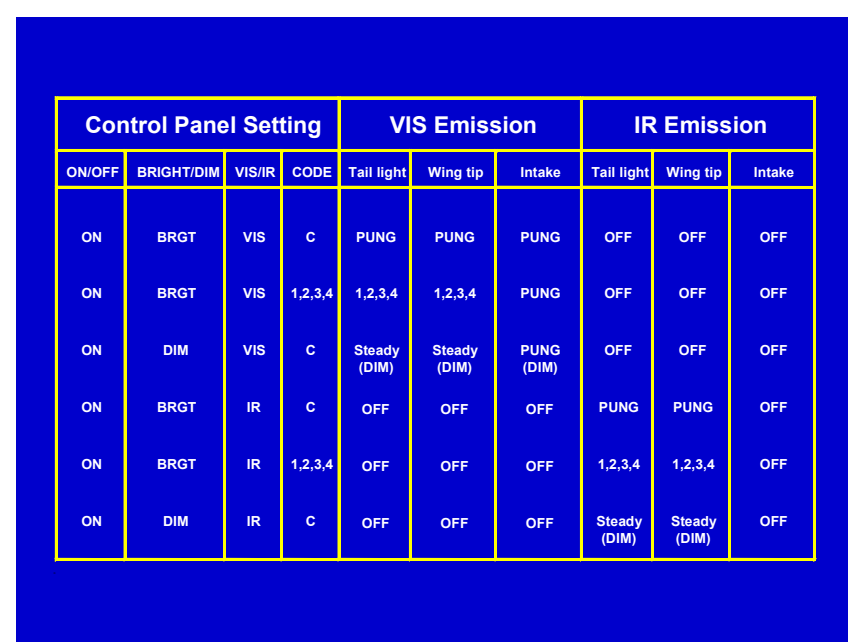

Figure 8: External lighting system functions.

The great majority of the TORNADO IDS/ECR cockpit displays, control panels and lights were modified by filtering or substituting the existing light sources, in order to obtain NVG compatible emissions. Also the aircraft external lights were modified, introducing an NVG friendly (IR emission) functional mode, and adding new functionalities in to the already existing visible lights. The new functionalities incorporated into the aircraft external lighting system are described in figure 8 . 
Citation: Sabatini R, Richardson MA, Cantiello M, Toscano M, Fiorini P, et al. (2013) Night Vision Imaging Systems Development, Integration and Verification in MilitaryFighter Aircraft. J Aeronaut Aerospace Eng 2: 106. doi:10.4172/2168-9792.1000106

Page 5 of 12

Particularly, a new control box was installed in the cockpit allowing the pilot selection of the various external lights functional modes. Five different codes, all square wave in nature (codes 1,2, 3, 4 and $\mathrm{C}$ in figure 4), were programmable in the control box (using an EPROM). One of these codes was programmed with equal on and off times, while the other codes were programmed according to aircrew requirements, selecting code sequences with flash repetition frequencies and flash durations well discernible in flight.

During the flight test activities, after introducing a large number of modification into the TORNADO IDS/ECR front and rear cockpits, it was observed that certain areas of the front/rear main instrument panels and of the front/rear left and right consoles were not sufficiently illuminated by self-contained and/or general purpose cockpit lighting. Therefore, it was decided to test a 'finger light' both in the front and in the rear cockpits. The finger light FINGERSTAR (P/N 4790-NF-01A) used in the trials had both IR and visible emissions available, selectable by the operator using a finger-switch located on an adjustable (left/right hand) switching rail.

\section{Test and Analysis Methods}

An innovative test matrix was used for assessing the operational suitability and effectiveness of the various modifications implemented in the cockpit (Figure 9). Particularly, both flight safety and operational effectiveness/suitability of the NVIS configuration were considered in the test matrix, allowing a direct correlation between the flight test rating criteria and the standard evaluation rating scale used by RSV. This approach was applied both to the single modified items under test (displays, lights, panels, etc.), and to the overall cockpit NVIS configuration.

Modified aircraft external lights (both VIS and IR modes) were tested in formation flights (chase aircraft), performing the following tasks:

- Tactical Rejoin

- Fighting Wing

- Close and Battle Formation

- Air-to-air Refueling

Also important was definition of criteria for Pilot and WSO workload

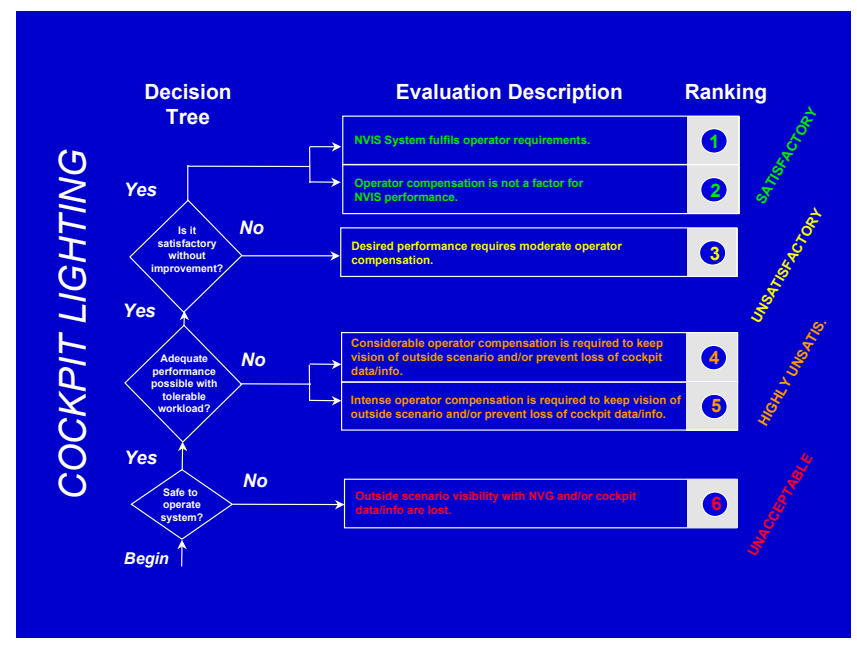

Figure 9: Cockpit evaluation test matrix.

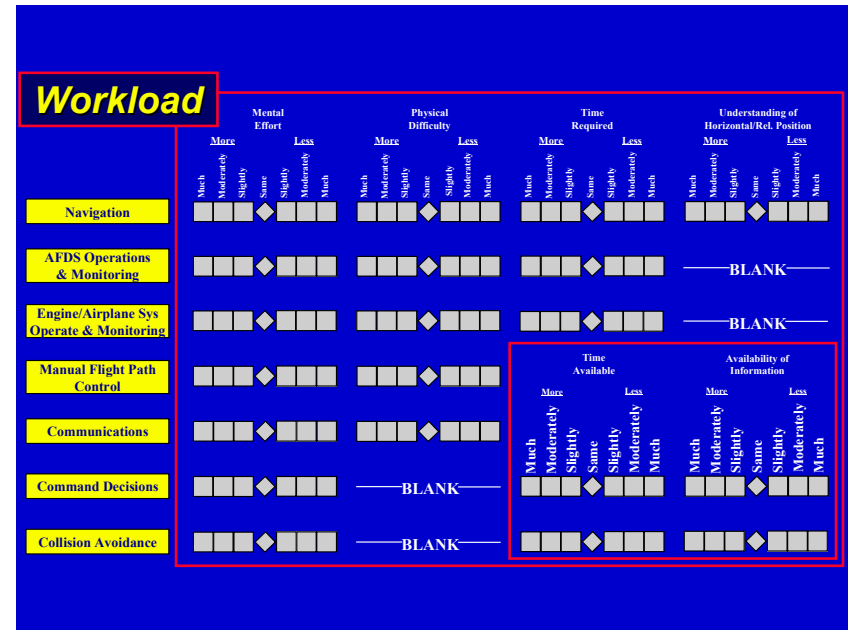

Figure 10: Workload evaluation matrix.

assessment during the accomplishment of various operational tasks during NVG missions (Figure 10). Particularly, a workload evaluation matrix was implemented that allowed identification of the workload levels associated with the various Pilot and WSO operational tasks during real missions. These included ferry flights, attack, formation flights and tactical evasive/escape manoeuvres. The operational tasks considered were the following:

- Navigation

- Automatic Flight Director System (AFDS) operation and monitoring

- Engine/airplane systems operation and monitoring

- Manual flight path control

- Communications

- Command decisions

- Collision avoidance

For each of the above tasks performed on the TORNADO NVG configuration, the levels of mental effort and physical difficulty, together with time required for the specific tasks and the understanding of horizontal/vertical position (spatial orientation) during execution of the tasks, were compared with the respective levels/values found for the standard TORNADO aircraft. Furthermore, the specific technical and operational elements required for evaluating the modified helmets were identified, allowing an exhaustive comparative evaluation of the two proposed solutions (i.e., HGU-55P and HGU-55G modified helmets). These elements included: measurement of the available FOV and calculation of the Projected FOV Area Reduction (PFAR), weight/ balance, comfort and stability, crew fatigue in low and high dynamics flights. Furthermore, the NVG connection/disconnection devices were tested performing high dynamics manoeuvres (with NVG both in the up-locked and down-locked positions).

In order to assess the operational suitability of the modified HGU55/P and HGU-55/G helmets, the related test activities focused on the following aspects:

- Measurement of the available Field-of-View (FOV) with minimum eye-relief 
Citation: Sabatini R, Richardson MA, Cantiello M, Toscano M, Fiorini P, et al. (2013) Night Vision Imaging Systems Development, Integration and Verification in MilitaryFighter Aircraft. J Aeronaut Aerospace Eng 2: 106. doi:10.4172/2168-9792.1000106

Page 6 of 12

- Determination of the minimum Projected Area FOV reduction (P-FOV)

- NVG helmets fitting and stability

- Clearance with a/c structure (NVG up-locked and down-locked)

- Fatigue in low dynamics flight

- Fatigue in maneuvering flight

- Possible use of protection visors

The spatial resolutions obtainable with the F4949 visors in the various sectors of the TORNADO canopy (normal sectors for external clearing), were also measured. This was done by using the USNTPS 20/20-20/70 resolution bars method [3]. Particularly, a resolution table was prepared (Figure 11), composed of 16 groups of bars with dimensions and spacing corresponding to visual acuities between 20/70 and 20/20. The resolution bars table was illuminated with an artificial light source reproducing typical night illumination conditions.

During a ground test, using the bars target shown in figure 13, together with the low illumination lamp, the spatial frequencies (cycle/mrad) corresponding to various 2-D discrimination levels were determined for the F4949 system used on TORNADO, in the various sectors of the aircraft canopy. Using these experimental data it was possible to calculate the detection, recognition and identification ranges of the NVG system, for targets of given aspect dimensions located in certain regions of the Pilot and WSO external clearing scanning patterns.

Before executing the on-board ground tests, a preliminary session were performed by the same aircrews (with NVG) positioned on the ground at a distance of 20 feet from the resolution table (illuminated by the low illumination lamp). In this condition, the groups of bars resolved were annotated. Also during successive the on-board session, the distance between the Pilot/WSO Reference Eye Positions (REP's) and the bars target was maintained to exactly 20 feet, and the resolution table was rotated about the REP's as shown in figure 12. Particularly, the following Pilot/WSO sectors were considered:

- Max Rear (Field-of-Regard limit)

- Lateral Sector $90^{\circ}$

- Lateral Sector $60^{\circ}$

- Lateral Sector $15^{\circ}-30^{\circ}$

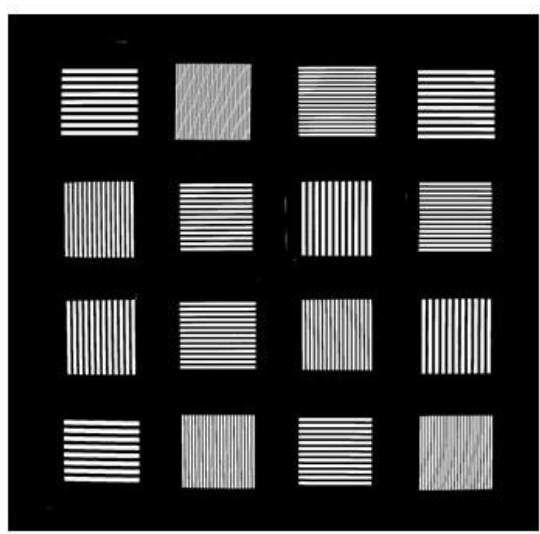

Figure 11: Resolution table (20/70-20/20).

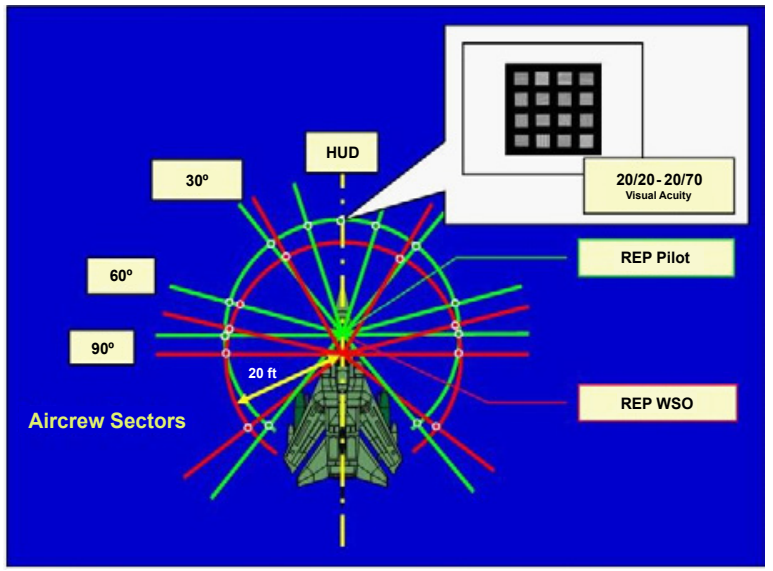

Figure 12: Geometry of resolution ground tests.

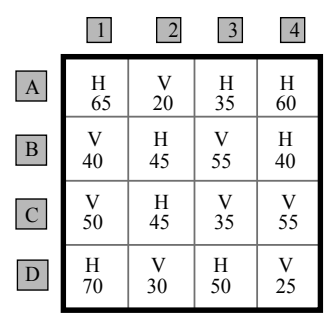

\begin{tabular}{llllll}
\hline & $\mathrm{C}$ & $\mathrm{B}$ & $\mathrm{A}$ \\
\hline
\end{tabular}

\begin{tabular}{|c|c|c|c|l|}
\hline $\mathrm{V}$ & $\mathrm{H}$ & $\mathrm{H}$ & $\mathrm{V}$ & 1 \\
70 & 50 & 40 & 65 & 1 \\
\hline $\mathrm{H}$ & $\mathrm{V}$ & $\mathrm{V}$ & $\mathrm{H}$ & 2 \\
30 & 45 & 45 & 20 & \\
& \\
$\mathrm{~V}$ & $\mathrm{H}$ & $\mathrm{H}$ & $\mathrm{V}$ & 3 \\
50 & 35 & 55 & 35 & \\
\hline $\mathrm{H}$ & $\mathrm{H}$ & $\mathrm{V}$ & $\mathrm{V}$ & \\
25 & 55 & 40 & 60 & 4 \\
\hline
\end{tabular}
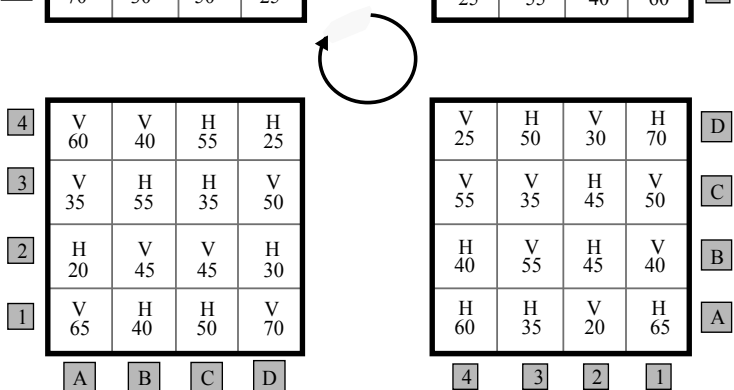

Figure 13: Resolution table positions for ground tests.

\section{- Pilot HUD $\left(0^{\circ}-15^{\circ}\right)$}

In each relevant position, the resolution target was rotated in four different positions as shown in figure 13. In each case, the Pilot/WSO abilities to resolve the various groups of bars were recorded.

NVG range performance predictions require a mathematical model that describes the eye/brain image interpretation process. Unlike the response of an electronic circuit, the response of a human observer cannot be directly measured but only can be inferred by many visual psychological experiments. The lowest level of discrimination is a distinction between something and nothing. The final level is the precise identification and description of a particular object. Between these two extremes lay a continuum of discrimination levels. In the late fifties, Johnson studied image intensifiers discrimination performance at the US Army Engineering and Research Laboratories [1]. He arbitrarily divided visual discrimination into four categories: detection, orientation, recognition, and identification. Johnson's results allowed correlating detectability with the sensor threshold bar pattern resolution (Table 1). In Johnson's work, the (angular) spatial frequency (SF) is defined as:

$$
S F=\frac{R_{T}}{W_{1 c}}
$$


Citation: Sabatini R, Richardson MA, Cantiello M, Toscano M, Fiorini P, et al. (2013) Night Vision Imaging Systems Development, Integration and Verification in MilitaryFighter Aircraft. J Aeronaut Aerospace Eng 2: 106. doi:10.4172/2168-9792.1000106

Page 7 of 12

\begin{tabular}{|c|c|c|}
\hline $\begin{array}{c}\text { Discrimination } \\
\text { level }\end{array}$ & Meaning & $\begin{array}{c}\text { Cycles across minimum } \\
\text { dimension }\end{array}$ \\
\hline Detection & $\begin{array}{c}\text { An object is present (object versus } \\
\text { noise) }\end{array}$ & $1.0 \pm 0.025$ \\
\hline Orientation & $\begin{array}{c}\text { The object is approximately } \\
\text { symmetrical or unsymmetrical and } \\
\text { its orientation may be discerned } \\
\text { (side view versus front view) }\end{array}$ & $1.4 \pm 0.35$ \\
\hline Recognition & $\begin{array}{c}\text { The class to which the object } \\
\text { belongs (e.g., tank, truck, man) }\end{array}$ & $4.0 \pm 0.80$ \\
\hline Identification & $\begin{array}{c}\text { The object is discerned with } \\
\text { sufficient clarity to specify the type } \\
\text { (e.g., T-52 tank, friendly jeep) }\end{array}$ & $6.4 \pm 1.50$ \\
\hline
\end{tabular}

Table 1: Summary of Johnson's experimental results.

\begin{tabular}{|c|c|c|}
\hline $\begin{array}{c}\text { Discrimination } \\
\text { level }\end{array}$ & Meaning & $\begin{array}{c}\text { Cycles across min. } \\
\text { dimension } \mathbf{( N}_{\mathbf{5 0}} \mathbf{)}\end{array}$ \\
\hline Detection & An object is present & 1.0 \\
\hline Recognition & The class to which the object belongs & 4.0 \\
\hline Identification & $\begin{array}{c}\text { The object is discerned with sufficient } \\
\text { clarity to specify the type }\end{array}$ & 8.0 \\
\hline
\end{tabular}

Table 2: Current industry criterion for $1-\mathrm{D}$ discrimination (50\% probability level).

\begin{tabular}{|c|c|}
\hline Probability of discrimination & Multipler $\mathbf{F}_{\mathbf{m}}$ \\
\hline 1.00 & 3.0 \\
\hline 0.95 & 2.0 \\
\hline 0.80 & 1.5 \\
\hline 0.50 & 1.0 \\
\hline 0.30 & 0.75 \\
\hline 0.10 & 0.50 \\
\hline 0.02 & 0.25 \\
\hline 0 & 0 \\
\hline
\end{tabular}

Table 3: Discrimination cumulative probability.

Where:

$\mathrm{R}_{\mathrm{T}}=$ sensor-to-target range;

$\mathrm{W}_{1 \mathrm{c}}=$ width of one cycle of target,

And the 'cycle' is defined as the sum of one bar and one space on the reference target. Johnson applied the number of cycles across the target minimum dimension, without regard to the orientation of the minimum dimension (his image intensifier imagery was radially symmetrical and therefore it was reasonable for him to ignore the bar orientation). Johnson's approach, known as the equivalent bar pattern approach, became the foundation for the discrimination methodology used today.

Successive studies and tests performed at the US Army Night Vision Laboratories and by industry suggested modifications to the values originally found by Johnson. Table 2 provides the current industry standard for one-dimensional target discrimination [2]. Orientation is a less popular discrimination level. Because current standards are based upon Johnson's work, they are labelled as the Johnson criterion though they are not the precise values found by him.

The Johnson criterion provides an approximate measure of the $50 \%$ probability of discrimination. Results of several tests provided the cumulative probability of discrimination or target transfer probability function (TTPF). The TTPF can be used for all discrimination tasks by simply multiplying the $50 \%$ probability of performing the task $\left(\mathrm{N}_{50}\right.$ in table 2) by the appropriate TTPF multiplier in table 3 [2].

For instance, the probability of $95 \%$ recognition is $2 \mathrm{~N}_{50}=2(4)=8$ cycles across the target minimum dimension. Similarly, the cycles required for detection, recognition and identification with a probability level of $80 \%$ are 1.5, 6 and 12 respectively. An empirical fit to the data provides [3]:

$$
P(N)=\frac{\left(\frac{N}{N_{50}}\right)^{E}}{1+\left(\frac{N}{N_{50}}\right)^{E}}
$$

Where:

$$
E=2.7+0.7 \cdot\left(\frac{N}{N_{50}}\right)
$$

Visual psychophysical experiments suggest that the eye response follow a log-normal distribution [4]. The probability density function follows:

$$
p(N)=\frac{1}{\sqrt{2 \pi} \cdot \log (\sigma)} \cdot e^{-\frac{1}{2}\left[\frac{\log (N)-\log \left(N_{50}\right)}{\log (\sigma)}\right]^{2}}
$$

Where $\log (\sigma)=0.198$. The cumulative probability is:

$$
P(N)=\int_{0}^{\log N} p(N) d \log (N)
$$

Both the empirical fit of eq (3) and the log-normal approach (based upon a physically plausible foundation) of eq (5) provide similar numerical results. As clutter increases, the ability to discern a target decreases. To account for this reduced capability, $\mathrm{N}_{50}$ must increase. Most studies have broadly categorized clutter into high, moderate and low regions, and defined the signal-to-clutter ratio (SCR) as:

$$
S C R=\frac{\max t \arg \text { et } \text { value }- \text { background mean }}{\sigma_{\text {clutter }}}
$$

Where:

$$
\sigma_{\text {clutter }}=\sqrt{\frac{1}{N} \sum_{i=1}^{N} \sigma_{i}^{2}}
$$

And $\sigma_{i}$ is the rms value of the pixel values in a square cell that has side dimensions of approximately twice the target minimum dimension. The scene is composed of $\mathrm{N}$ adjoining cells. The use of adjoining cells introduces a spatial weighting factor that is similar to the spatial integration performed by the eye/brain process. Clutter sizes that are equal to the object size weigh more heavily in this calculation.

The results are presented in table 4 [5].

Field experiments demonstrated that the Johnson detection criterion applies to a "general medium to low clutter" environment. Therefore, the $50 \%$ probability of detection in table 4 where normalized in moderate clutter to one cycle. These experimental findings roughly follow the empirical TTPF of eq (2). It is convenient to use 0.5, 1.0 and 2.5 as a multiplier $\left(\mathrm{F}_{\mathrm{d}}\right)$ to $\mathrm{N}_{50}$ for low, moderate, and high clutter environments respectively.

In order to obtain the two-dimensional discrimination levels required in a $2-\mathrm{D}$ performance prediction model, each value in the 
Citation: Sabatini R, Richardson MA, Cantiello M, Toscano M, Fiorini P, et al. (2013) Night Vision Imaging Systems Development, Integration and Verification in MilitaryFighter Aircraft. J Aeronaut Aerospace Eng 2: 106. doi:10.4172/2168-9792.1000106

Page 8 of 12

\begin{tabular}{|c|c|c|c|}
\hline \multirow{2}{*}{$\begin{array}{c}\text { Probability of } \\
\text { detection }\end{array}$} & \multicolumn{3}{|c|}{ Multiplier $F_{d}$} \\
\hline & $\begin{array}{l}\text { Low Clutter } \\
\text { SCR }>10\end{array}$ & $\begin{array}{l}\text { Moder. Clutter } \\
1<S C R<10\end{array}$ & $\begin{array}{l}\text { High Clutter } \\
\text { SCR }<1\end{array}$ \\
\hline 1.0 & 1.7 & 2.8 & ** \\
\hline 0.95 & 1.0 & 1.9 & ** \\
\hline 0.90 & 0.90 & 1.7 & $7.0^{*}$ \\
\hline 0.80 & 0.75 & 1.3 & 5.0 \\
\hline 0.50 & 0.50 & 1.0 & 2.5 \\
\hline 0.30 & 0.30 & 0.75 & 2.0 \\
\hline 0.10 & 0.15 & 0.35 & 1.4 \\
\hline 0.02 & 0.05 & 0.1 & 1.0 \\
\hline 0 & 0.0 & 0.0 & 0.0 \\
\hline
\end{tabular}

** No data available. * Estimated

Table 4: TTPF when clutter is present.

\begin{tabular}{|c|c|c|}
\hline Discrimination level & Meaning & $\begin{array}{c}\text { Cycles across minimum } \\
\text { dimension } \mathbf{( N}_{\text {50-2D }} \mathbf{)}\end{array}$ \\
\hline Detection & An object is present & 0.75 \\
\hline Recognition & $\begin{array}{c}\text { The class to which the } \\
\text { object belongs }\end{array}$ & 3.00 \\
\hline Identification & $\begin{array}{c}\text { The object is discerned } \\
\text { with sufficient clarity to } \\
\text { specify the type }\end{array}$ & 6.00 \\
\hline
\end{tabular}

Table 5: Discrimination levels for the 2-D model (50\% probability level).

one-dimensional criteria (Table 5) is multiplied by 0.75 . The results are presented in table 5.

The US Night Vision Laboratory Static Performance Model [6] uses the minimum dimension (1-D), whereas most 2-D models refer to the object critical dimension [7]:

$$
h_{c}=\sqrt{W_{T G T} \times H_{T G T}}
$$

Where $\mathrm{W}_{\mathrm{TGT}}$ and $\mathrm{H}_{\mathrm{TGT}}$ are the horizontal and vertical object dimensions. In this case, the number of cycles used for range performance calculations is that associated to the critical dimension $h_{c^{*}}$

In conclusion, our 2-D range performance prediction model is summarized by the following equations:

$$
\begin{aligned}
& R=\frac{h_{c}}{\left(N_{50-2 D} \times F_{d}\right)} \times S F \text { for detection } \\
& R=\frac{h_{c}}{\left(N_{50-2 D} \times F_{m}\right)} \times S F \text { for recognition and identification }
\end{aligned}
$$

where

$\mathrm{R}=$ predicted slant range;

$\mathrm{h}_{\mathrm{c}}=$ target critical dimension;

$\mathrm{S}_{\mathrm{F}}=$ measured spatial frequency;

$\mathrm{N}_{50-2 \mathrm{D}}=$ cycles required for detection, recognition and identification;

$\mathrm{F}_{\mathrm{m}}, \mathrm{F}_{\mathrm{d}}=$ multipliers for the various discrimination levels.

\section{Test Results}

The activities on TORNADO IDS and ECR both included various ground test sessions and a total of eighteen flight test sorties (7 night flights and 2 day flights for each aircraft type). RSV and Litton Precision Products were responsible of coordinating and conducting the installation activities of the internal and external lights. Particularly, an iterative process was established, allowing an in-site rapid correction of the major deficiencies encountered during the ground and flight test sessions. Both single-ship (day/night) and formation (night) flights were performed, shared between the Test Crews involved in the activities (Test Pilots/WSOs), allowing for a redundant examination of the various test items by all participants.

The technical results of the activity were very satisfactory. Particularly, the internal lighting compatibility problems were progressively mitigated by incorporating modifications both in the front and rear cockpits at the various stages of the development test program. This process allowed a considerable enhancement of the TORNADO cockpits NVIS configurations, giving a good medium-high level NVG operational capability to the aircraft. The Air Force Operational Certifications for both the IDS and ECR aircraft configurations were achieved by 2002 . Figure 14 shows the initial and final results of the overall cockpit evaluation.

All external lighting modifications incorporated into the aircraft where satisfactory. Particularly, all medium-high level flight tasks required were performed successfully, after an adequate level of aircrew training. Close formation flights were indeed some of the most demanding tasks during NVG operations, requiring an appropriate level of aircrew training in order to estimate other aircraft distance, attitude and speed (dept/distance perception is severely degraded by NVG).

The workload assessment also gave encouraging results, demonstrating that the modifications of the aircraft interior and exterior lighting increased the levels of Pilot/WSO situational awareness and therefore their ability to perform operational tasks in night conditions. Particularly, medium-high level navigation and communications tasks where performed without a significant increase of aircrew workload, while the increase of workload experienced in AFDS/Engine/ Airplane Systems operation and monitoring was counterbalanced by the substantial reduction of workload experienced in manual flight path control, command decisions, and collision avoidance tasks (e.g., formation flights). Again, it was readily apparent during the tests, that aircrew training was the key to increase flight safety and operational effectiveness in NVG operations.

The results of the NVG-helmets ergonomic evaluation are summarized in figure 15 . The modified HGU-55/G helmet was heavier

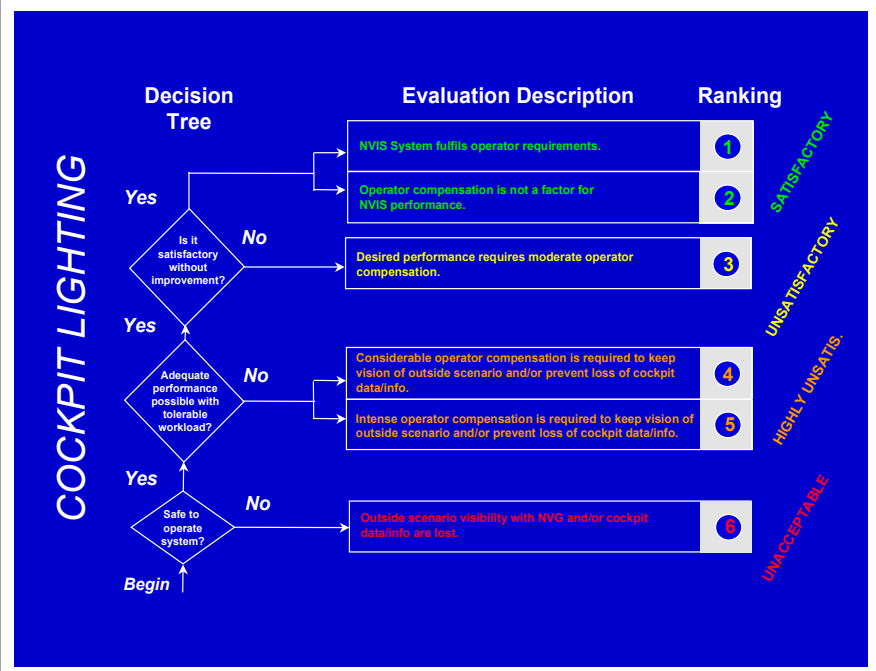

Figure 14: Results of the cockpit evaluation. 
Citation: Sabatini R, Richardson MA, Cantiello M, Toscano M, Fiorini P, et al. (2013) Night Vision Imaging Systems Development, Integration and Verification in MilitaryFighter Aircraft. J Aeronaut Aerospace Eng 2: 106. doi:10.4172/2168-9792.1000106

Page 9 of 12

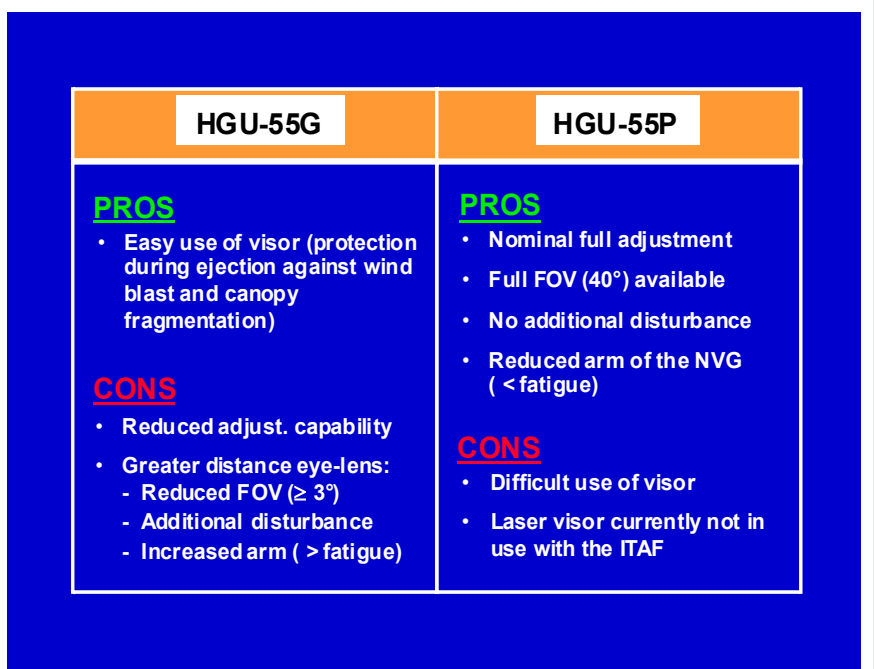

Figure 15: Results of helmets ergonomic evaluation

\begin{tabular}{|c|c|c|c|c|c|}
\hline \multicolumn{2}{|c|}{ FOV } & Diff. FOV & \multicolumn{2}{|c|}{ PFAR } & Diff. PFAR \\
\hline HGU-55P & HGU-55G & 1.98 & HGU-55P & HGU-55G & $10.14 \%$ \\
\hline 39.19 & 37.21 & & $4.30 \%$ & $14.44 \%$ & \\
\hline
\end{tabular}

Table 6: FOV e PFAR measurements.
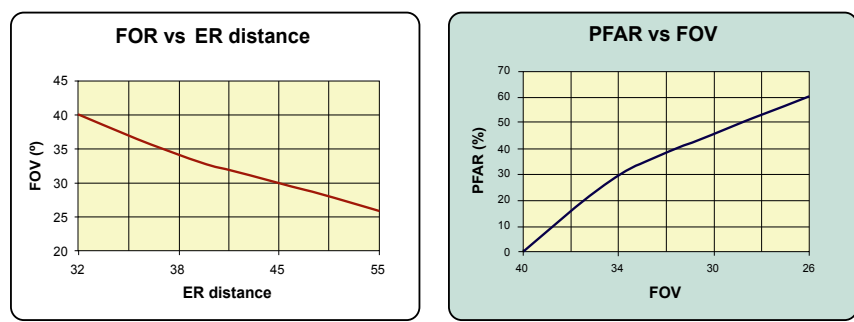

Figure 16: FOV vs. ERD and PFAR vs. FOV curves.

and less stable/balanced than the HGU-55/P helmet, and also gave a reduced NVG FOV due to increased eye-relief. However, the HGU55/P helmet was not suitable for operational use, due to difficulties in installing and removing the clear/laser protection visors during night operations with NVG (flying with protection visors is required on TORNADO to protect the aircrew, in case of ejection, against windblast and canopy fragmentation).

Table 6 shows the experimental data relative to the NVG FOV and PFAR, obtained with the HGU-55/G and HGU-55/P modified helmets, used by an operator with average percentiles, wearing a medium size helmet and a medium size oxygen mask (similar results were obtained with operators having different percentiles).

Compared to the $40^{\circ}$ nominal FOV of the F43949 system, it is evident that there was a decrease in FOV of about $0.8^{\circ}$ for the HGU$55 / \mathrm{P}$ helmet, and of $2.8^{\circ}$ for the HGU-55/G helmet (i.e., the HGU-55/P helmet gives a $2^{\circ}$ increase of FOV due to a reduced eye-relief). With the same operator, the PFAR (i.e., reduction of imaged scene area covered by the NVG), was about $4 \%$ for the HGU-55/P and about $14 \%$ for the HGU-55/G. Therefore, there was a difference of about $10 \%$ in the area covered by the NVG between the two helmets.

Based on the F4949 design data (provided by ITT Night Vision), figure 16 shows the FOV calculated as a function of the eye-relief distance and the PFAR vs. FOV curve.

The experimental PFAR data (Figure 17) were essentially coherent with the theoretical calculations. It is worth to underline that an ERD increase of $1 \mathrm{~mm}$ determines a $1^{\circ}$ reduction in FOV, and an increase of the PFAR of about $5 \%$. Compared to the ideal case of $\mathrm{FOV}=40^{\circ}$, this would equate to a $20 \%$ reduction of the area covered by the NVG for the HGU-55/G helmet, and of about $10 \%$ for the HGU-55/P helmet

Based on visual acuity measurements results, the NVG detection, recognition and identification range performances were calculated using equations (9) and (10), for different types of targets. Particularly, the detection/recognition/identification range performances were calculated with $80 \%, 90 \%$ and $100 \%$ probability levels. Furthermore, the detection performances $(80 \%, 90 \%$ and $100 \%$ probability) were also calculated in low, medium and high clutter conditions [8]. Examples of the results obtained are shown in figure 18.

\section{Lessons Learned}

The Human Factors risks in NVG operations are directly related to the quality of the interior and exterior aircraft lighting, the quality of aircrew training and the ability to detect and quantify under NVG. The most important technical and operational lessons learned during the TORNADO NVG flight test activities were the following:

- Unaided readability is just as important as NVG compatibility. NVG flight can be regarded as 'visually aided' instrument flight.

- A poor installation can spoil a good modification design (e.g., incompatible light leaks).

- Daylight readability may be more difficult after NVG modifications. Suppression of warning/caution indicators within the NVG FOV has to be avoided.

- The same design rationale for standard lighting applies to NVIS lighting.

- Standard lighting cannot be turned down enough to be NVG compatible.

\section{Experimental PFAR}

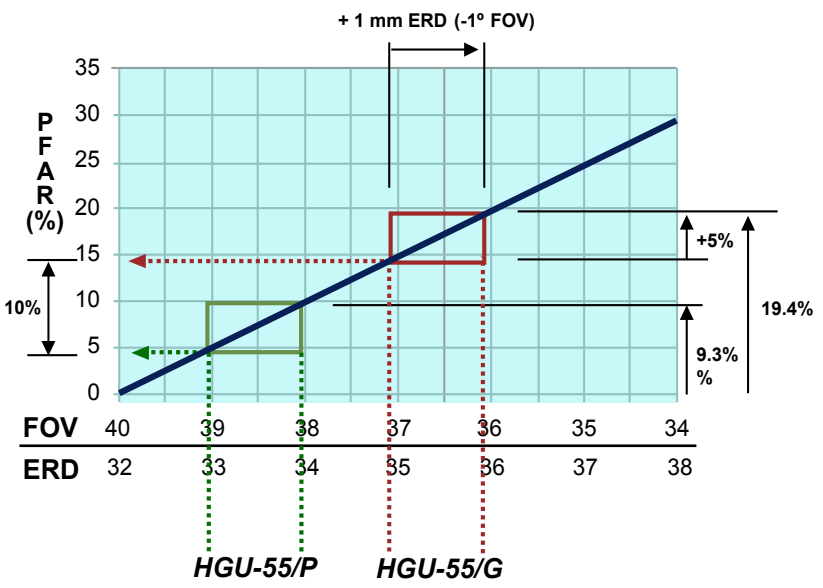

Figure 17: Percent variation of the PFAR as a function of ERD and FOV. 
Citation: Sabatini R, Richardson MA, Cantiello M, Toscano M, Fiorini P, et al. (2013) Night Vision Imaging Systems Development, Integration and Verification in MilitaryFighter Aircraft. J Aeronaut Aerospace Eng 2: 106. doi:10.4172/2168-9792.1000106

Page 10 of 12

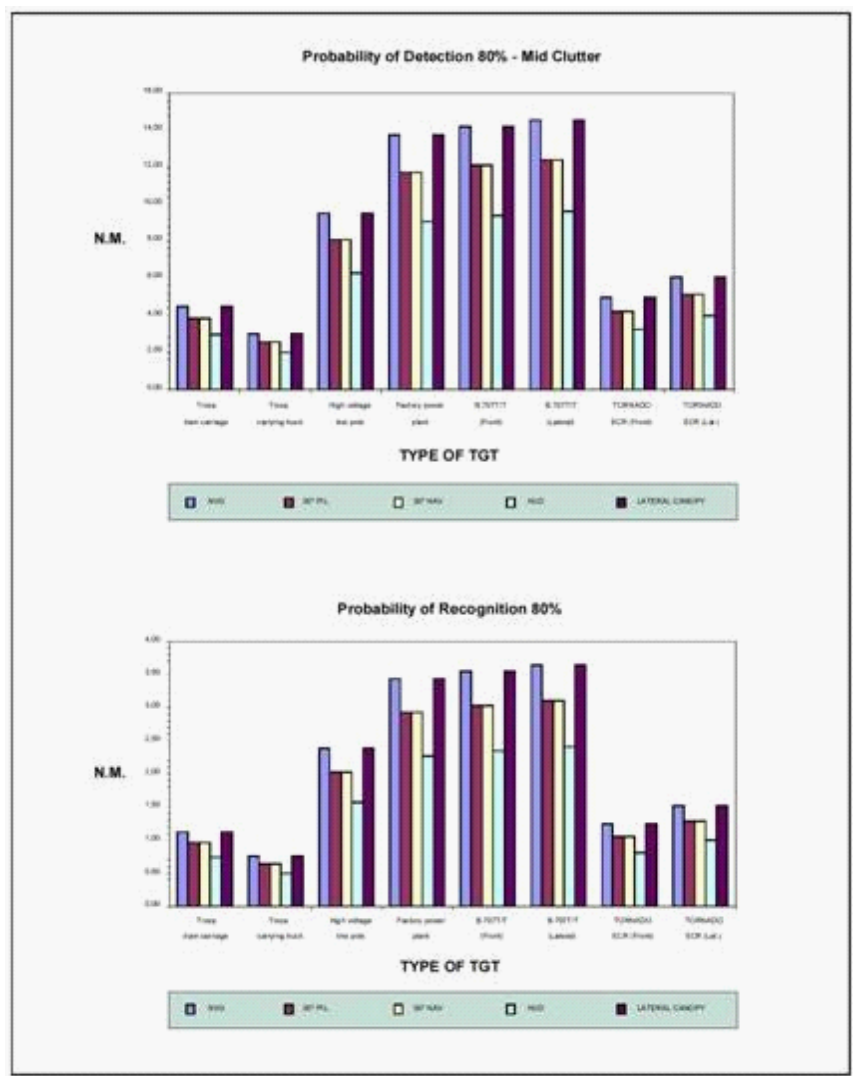

Figure 18: Results of NVG range performance calculations.

- Partial modification is usually not successful. NVGs used for long periods may result in increased workload for aircrew.

- Properly designed NVIS lighting is usually superior to lighting it replaces. Particularly, it reduces reflections on canopies; it makes instruments easier to read at lower brightness levels, and reduces eye fatigue.

\section{Conclusions and Further Developments}

In this paper we have described the development and testing activities conducted on the Italian TORNADO IDS/ECR in order to confer a medium-high level NVG operational capability to the aircraft. The TORNADO development activities, addressing the aircraft interior/ exterior lighting and the helmet modifications (NVG integration), were conducted by RSV and supported by industry (Litton Presion Products). Also the ground and flight test activities were conducted by RSV, with participation of industry to the test flights (Alenia).

Particularly important for RSV was the clear identification of the technological alternatives available for aircraft modifications, as well as the definition of suitable test methods for both internal and external lighting evaluation. Also very important was the adoption of appropriate NVG performance analysis models, which leaded to the development of a standard PC based data analysis tool.

The technical results of the TORNADO NVG activities were very satisfactory. Particularly, the internal lighting compatibility problems were progressively mitigated by incorporating modifications both in the front and rear cockpits at the various stages of the development test program. This process allowed a considerable enhancement of the
TORNADO cockpits NVIS configurations, giving a good medium-high level NVG operational capability to the aircraft.

The workload assessment also gave encouraging results, demonstrating that the modifications of the aircraft interior and exterior lighting increased the levels of Pilot/WSO situational awareness and therefore their ability to perform operational tasks in night conditions. However, it was readily apparent during the tests, that aircrew training was the key to increase flight safety and operational effectiveness in NVG operations.

The NVG-helmets tests allowed a comprehensive verification of the ergonomic and technical elements in favor or against each of the proposed solutions (i.e., modified HGU-55/G and HGU-55/P helmets). Overall, the HGU-55/P helmet was rejected due to difficulties in installing and removing the clear/laser protection visors during night operations, while the modified HGU-55/G was selected for TORDADO IDS/ECR operations (although not fully satisfactory).

In conclusion, a considerable experience was gained during the TORNADO NVG activities and further developments were launched in this area, taking advantage of the technical and operational lessons learned, to increase the ITAF aircraft operational capability and safety. Further developments include the Alenia internal/external lighting design for the Italian TORNADO "Mid Life Update" (MLU) and various other Air Force programs, such as the AM-X aicraft internal/ external lights modification/testing and other activities addressing lowaltitude NVG operations with fast jets (e.g., TORNADO, AM-X, MB339CD). A major issue encountered is the safe ejection of aircrew with NVG and NVG modified helmets. Two options have been identified for solving this problem: modification of the current HGU-55 helmets and the design of a new helmet incorporating a reliable NVG connection/ disconnection device (i.e., a mechanical system fully integrated in the helmet frame), with embedded automatic disconnection capability in case of ejection. Other relevant issues to be accounted for in these new developments are the helmet dimensions and weight, the NVG usable FOV as a function of eye-relief distance, and helmet centre of gravity (moment arms) with and without NVG (impact on aircrew fatigue during training and real operational missions). A pictorial representation of the system initially proposed by Gentex and ITT Night Vision in order to match the Italian and German Air Forces TORNADO helmet requirements is shown in figure 19.

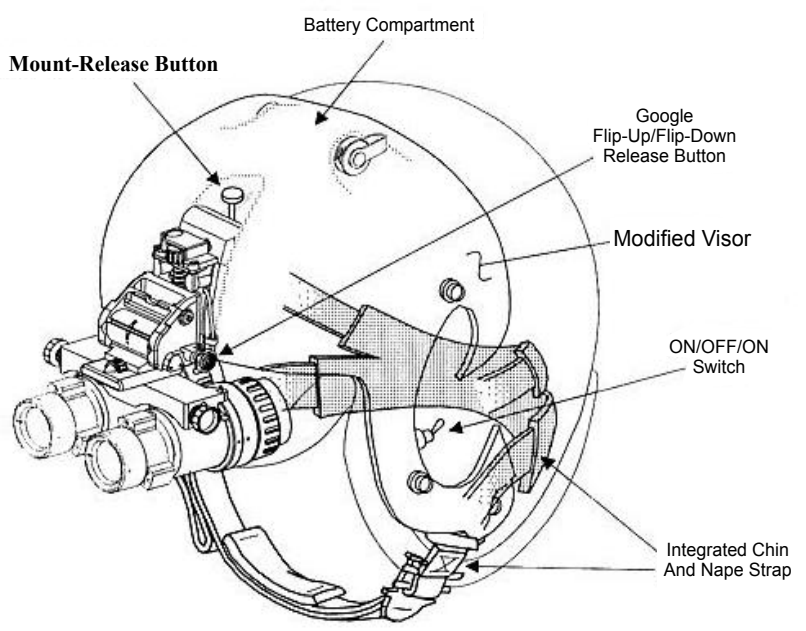

Figure 19: ITT/Gentex proposed NVG helmet for TORNADO 
Citation: Sabatini R, Richardson MA, Cantiello M, Toscano M, Fiorini P, et al. (2013) Night Vision Imaging Systems Development, Integration and Verification in MilitaryFighter Aircraft. J Aeronaut Aerospace Eng 2: 106. doi:10.4172/2168-9792.1000106

The ITAF requirements for a new helmet allowing a safe and practical usage of the F4949P NVG were established so that no restrictions were applied to the aircraft operational flight envelopes due to use of the NVG system. In order to achieve this, the new development should address the following main issues:

- maximise the operator's usage of the NVG performance

- maximise the balancing, stability and comfort of the new helmet

- maximise the level of safety (normal use and ejection)

The overall goals to be achieved in the development are the following:

- No modifications of the existing F4949P NVG system

- NVG usable in "up-locked" and "down-locked" positions

- Practical and safe connection/disconnection of the NVG/Adapter

- Maximum usage of the available NVG FOV

- No protrusions on the helmet

- No Helmet weight increase

- NVG-Adapter moment arm minimisation

- Maximum comfort and stability also under g's

- Use of helmet visors (inner clear/laser visor for NVG operations and dark outer visor for operations without NVG)

- Availability of documentation required for Helmet/Adaptor Qualification and Certification (i.e., System Performance Specification, System Design Documentation, and Development Test Reports)

The new developments shall not include modifications of the existing F4949P NVG system. Furthermore, the NVG should be usable both in 'up-locked' and 'down-locked' positions, without possibility of NVG disconnection in these positions from the Adapter-Helmet. Manual disconnection of the NVG from the Adapter-Helmet should be possible only in a dedicated 'intermediate' position. Self-disconnection during ejection should be guaranteed independently from the NVG position.

Connection and disconnection of the F4949P NVG, of the Helmet Adapter and of the NVG-Adapter block should be possible for the operator with a single action and using a single hand. Particularly, the entire NVG-Adapter block should be removable as one section (e.g., before ejection), the F4949P NVG should be separately removable from the Adapter-Helmet (e.g., for normal stowing of the NVG), and the adapter should be also separately removable from the Helmet (using the same device available for removal of the NVG-Adapter block). Additional detailed requirements are:

- During the initial phase of a seat-ejection (i.e., acceleration phase) the NVG-Adapter block should fall off the helmet without any action of the crew

- The modified Helmet-Adapter should allow usage of the maximum Field Of View (FOV) provided by F4949P NVG

- The Helmet should be free from significant protrusions. The Adapter block should be designed to minimise protrusions, as to allow a smooth surface of the Helmet-Adapter combination

- All efforts should be placed in order to minimise the weight of the modified Helmet. Particularly, it is desirable that the weight of the new helmet does not increase with respect to the current helmets and, if feasible, it should be reduced

- The moment arm of the NVG-Adapter block should be minimised, in order to obtain a balanced Helmet and to maximise the Helmet stability and fitting comfort

- The inner part of the helmet should be modified in order to enhance the helmet stability (also under g's) by using combined ChinNap Straps or other stability enhancing features

- The helmet should be equipped with two visors: an inner visor (i.e., clear visor or laser visor) and an outer visor (i.e., dark visor). The F4949P NVG system will be used with the inner visor down

Recent studies conducted by ITT-Night Vision and Gentex, in collaborationwith ITAF and the Italian MoDhave leaded to the NVGhelmet solutions shown in figures 19 and 20. Particularly, two different technical options were identified: one which is based on the HGU-55/G helmet (Figure 20) and another based on the HGU-55/P helmet (Figure 21).

\section{Acknowledgements}

The authors would like to acknowledge the valuable contribution given by Alenia and Litton Precison Products, during the TORNADO IDS/ECR NVG development and flight test activities. Great thanks go to the staff of RSV for strongly supporting NVIS programs. Last, but not least, the author wishes to thank the aircrews and

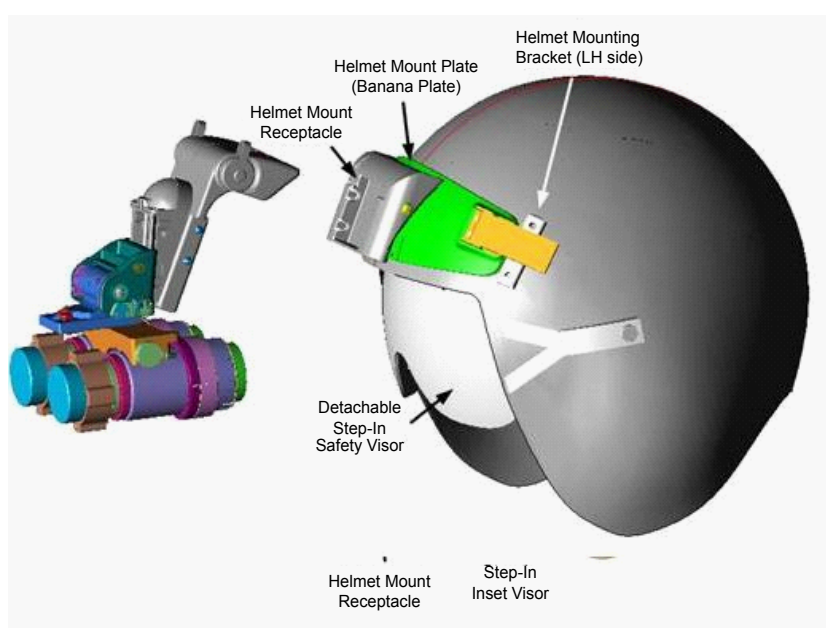

Figure 20: Proposed HGU-55/G NVG helmet.

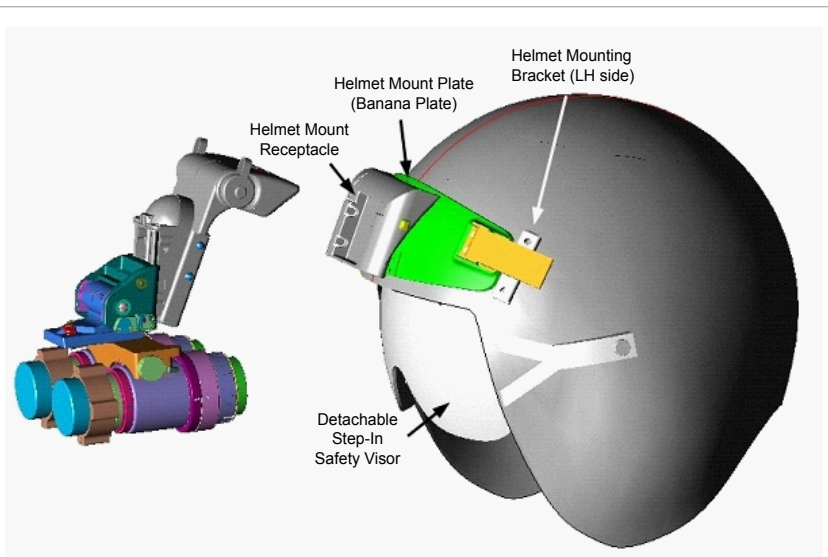

Figure 21: Proposed HGU-55/P NVG helmet. 
Citation: Sabatini R, Richardson MA, Cantiello M, Toscano M, Fiorini P, et al. (2013) Night Vision Imaging Systems Development, Integration and Verification in MilitaryFighter Aircraft. J Aeronaut Aerospace Eng 2: 106. doi:10.4172/2168-9792.1000106

technical personnel of the Alenia Flight Test Department. Thanks also go to all Air Force, Alenia, Litton, ITT and Gentex personnel not explicitly mentioned here, which supported in different ways the TORNADO NVG development programs. A shorter version of this paper was presented at the SPIE Photonics Europe 2012 Conference, held in Brussels in March 2012

\section{References}

1. Johnson J (1985) Analysis of Image Forming Systems. Proceedings of SPIE, USA.

2. Ratches JA (1976) Static Performance Model for Thermal Imaging Systems Opt Eng 15: 525-530.

3. Howe JD (1993) Electro-Optical Imaging System Performance Prediction. The Infrared \& Electro-Optical Systems Handbook. SPIE Press 57-116.
4. Gerald HC (1992) Applying the log-normal distribution to target detection. Proc SPIE 1689: 213-216.

5. Schmieder DE (1983) Detection Performance in Clutter with Variable Resolution. IEEE Transactions on Aerospace and Electronic Systems. AES-19: 622-630.

6. James RA, Walter LR, Luanne OP, Richard BJ, Thomas CW (1975) Night Vision Laboratory Static Performance Model for Thermal Viewing Systems. Army Electronics Command Fort Monmouth NJ.

7. Holst GC (2003) Electro-optical imaging system performance SPIE Press monograph. JCD Pub.

8. Roberto S, Mark RA, Maurizio C, Mario T, Pietro F, et al. (2012) Night vision imaging systems design, integration, and verification in military fighter aircraft. Proceedings of the SPIE, USA 